\title{
Emergency Tracheal Surgery for Adenoid Cystic Carcinoma: A Rare Entity
}

\author{
Farhan Ahmed Majeed1, Usama Zafar1, Ahmad Ali1, Umer Mehmood² and Mohammad Rashid Husnain
}

\begin{abstract}
Primary tumours of the trachea are rare. Adenoid cystic carcinoma (ACC) constitutes less than $1 \%$ of these tumours. They occasionally masquerade as asthma. We are presenting a case of a young female, who had been treated for 3 years as asthma. She presented in emergency with severe respiratory difficulty, stridor and decreasing saturation of $85 \%$ on room air. Two weeks back, she had a CT scan in a local hospital, but they did not notify the tracheal mass. Her clinical picture and CT scan helped us diagnose the case. She was treated in emergency with primary resection of tracheal mass and anastomosis. The tumor was diagnosed as ACC. Postoperative recovery was uneventful. Continuity of the trachea was restored and adjuvant radiotherapy was given.
\end{abstract}

Key Words: Adenoid cystic carcinoma, Tracheal resection, Primary anastomosis, Tracheal tumours.

\section{INTRODUCTION}

Primary tracheal tumours account for less than $1 \%$ of all malignancies. 1 Adenoid cystic carcinoma (ACC) of trachea accounts for $16 \%$ of the tracheal tree tumours, and stands second in tracheal tumours. It is one of the slow growing tumours. Primary resection of tumour and anastomosis followed by radiotherapy is the mainstay of treatment. We have a case of a young female who had been treated for 3 years as asthma, and presented in emergency department with severe dyspnea.

ACC is primarily a salivary gland tumour and it invades neural tissue. Submucosal glands in respiratory system give rise to this tunour.2,3 It is one of the slow growing tumors. It is known for local recurrence and later metastasis.

\section{CASE REPORT}

A 25-year woman patient, progressively developed difficulty in respiration during the last 3 years. She was labelled as asthmatic, and treated for it. One fine morning, she crashed in our emergency department with severe respiratory difficulty. She was anxious and irritable. On examination, she had audible stridor. Her oxygen saturation was around $85 \%$ on room air. She was resuscitated with bronchodilator nebulisation and oxygen support. She had a computerised tomography (CT) scan about two weeks ago. Study of CT scan showed a tracheal mass about 3 centimeter $(\mathrm{cm})$ below the vocal cords causing obstruction of $75 \%$ trachea

Department of Thoracic Surgeryl / General Surgery2,

Combined Military Hospital, Lahore, Pakistan

Correspondence: Dr. Usama Zafar, Department of Thoracic

Surgery, Combined Military Hospital, Lahore, Pakistan

E-mail: usamazafar0705@gmail.com

Received: March 05, 2018; Accepted: June 26, 2018
(Figure 1). The case was immediately discussed with the thoracic surgeon, who was operating on another patient. It was determined that the cause of respiratory difficulty and stridor is a mechanical obstruction by the mass and surgery was the option. It was decided that she would be best managed in operating theatre complex where better facilities of resuscitation and surgery are available. Thus, she was shifted to the operation theater for definite treatment / tracheostomy.

Flexible bronchoscope was not tolerated by the patient, so in operating room, she underwent rigid bronchoscopy, which showed a mass in trachea beyond four tracheal rings occupying $70 \%$ of the trachea. It was negotiated with bougie with guide wire passed, which was used to railroad the endotracheal tube (ETT) size 5. This secured the airway and it was decided to do definitive surgery.

Resection of tracheal tumour with primary end-to-end anastomosis was planned. A suprasternal collar incision was given on the neck. Thyroid isthmus was cut and thyroid lobes were retracted laterally with stay sutures. Trachea was opened below four tracheal rings. Tumour was involving seven tracheal rings (approximately $3.5 \mathrm{~cm}$ ) from $3^{\text {rd }}$ to $9^{\text {th }}$ ring. Digital mobilisation in pretracheal space posteriorly and up to carina and constant neck flexion were used for approximation of both tracheal ends to ensure tension-free anastomosis. Tracheal length containing tumour was cut. Figure 2 shows resected tracheal tumour. Cross-field ventilation was maintained in the distal tracheal end. Interrupted anastomosis with alternate vicryl $3 / 0$ and $4 / 0$ was done. Thyroid flap was superimposed over the trachea. Grillo's stitch was applied from chin to upper chest to ensure flexion postoperatively. Redivac drain was placed in the neck.

Postoperative recovery was uneventful. Tracheal anatomy was restored to normal and the patient was discharged with normal breathing and oxygen saturation. 


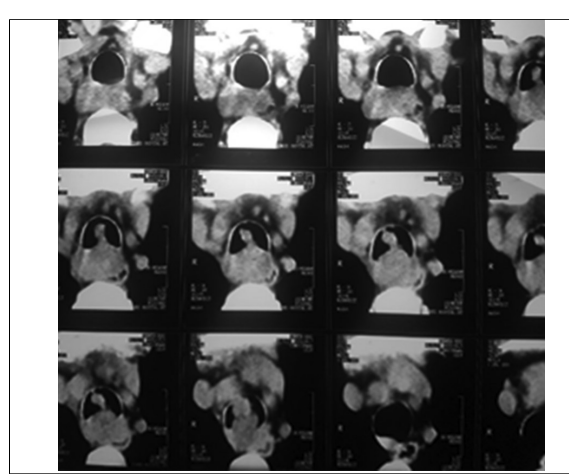

Figure 1: CT film showing mass in trachea

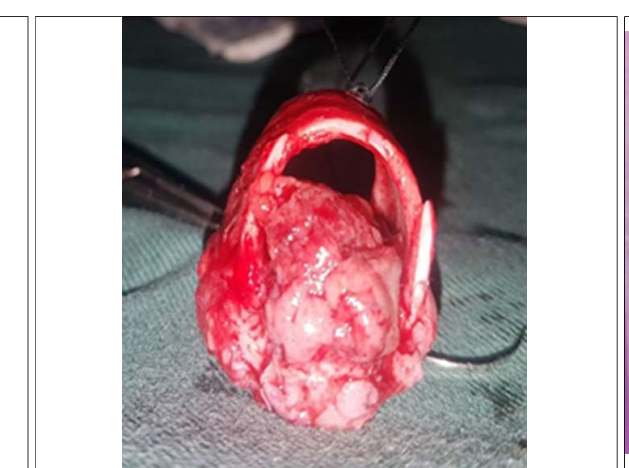

Figure 2: Specimen showing tumour involving the tracheal lumen.

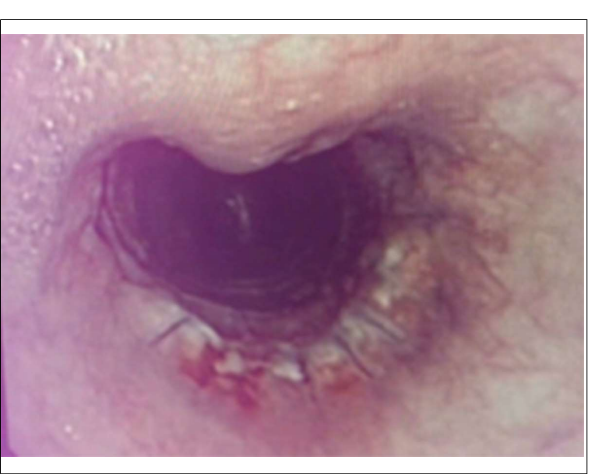

Figure 3: Post-op bronchoscopy showing normal continuity of trachea and anastomosis site showing stitches.
CT scan with 3D reconstruction of the tracheobronchial tree and flexible bronchoscopy was performed after surgery, which showed normal trachea. Figure 3 is postoperative bronchoscopy showing normal continuity of trachea, and anastomosis site showing the stitches.

Histopathology report showed it to be ACC moderately differentiated grade 2. Tumour was involving inferior tracheal margins. The tumor was CD117 positive and p63 negative. The case was presented at multidisciplinary meeting, where it was decided to further treat the patient with radiotherapy.

\section{DISCUSSION}

Tracheal tumours are rare and constitute less than $1 \%$ of all malignancies ( 0.1 person / 100,000 people). ACC is even rarer accounting for $16 \%$ of tracheal malignancies and is preceded by squamous cell carcinoma (SCC), which accounts for $80 \%$ of tracheal malignancies. 1 Tracheal tumours are usually slow growing. Secondary tracheal malignancies usually arise from surroundings such as esophagus, thyroid, lungs, and larynx. ${ }^{1}$

Insidious presentation is a hallmark of tracheal malignancies. They are usually treated as asthma, pneumonia or chronic obstructive pulmonary disease (COPD). Clinical presentation points towards upper airway obstruction. The suspicion arises with audible stridor and, if time and patient condition permits, is confirmed radiologically. Computerised tomography (CT) is the radiological investigation of choice. 1 Bronchoscopy, investigative modality of choice, is used for both diagnostic and therapeutic purposes, direct visualisation of mass, biopsy for tissue diagnosis and laser treatment. Conventional X-ray chest helps very little for the diagnosis. Intraoperative use of bronchoscopy helps to identify the exact site of tumour.2,3

Resection and anastomosis of tracheal tree pose some special challenges, which are unique for it. Maintenance of airway is very unique to resection of tracheal tree. Techniques have been developed for the satisfactory management of distal airway. There is jet ventilation, extra corporeal membranous oxygenation (ECMO), and percutaneous trans-sternal ventilation. Length of the trachea to be resected and maintaining tension-free anastomosis also pose a difficult problem. Various techniques are employed for this purpose. These include posterior and lateral mobilisation of trachea, neck flexion, digital mobilisation up to carina and laryngeal release. Up to half of the trachea can be resected safely by employing these techniques. Upper one-third tumours are approached transcervically using a collar incision. Neck incision along with sternotomy, either partial or complete, is used for middle-third tumours. Lower-third tumours are approached with right thoracotomy approach.

ACC is also called cylindroma. It spreads submucosally and may involve nearby lymph nodes. Lung metastases may also occur. ACC has a better prognosis compared to SCC. Five-year survival rate is $66-100 \%$ and 10 -year survival rate is $51-62 \% .1$ Primary resection and anastomosis followed by radiotherapy, and sometimes chemotherapy, are the mainstay of treatment. ${ }^{4}$ Other treatment options are radiotherapy and laser treatment. Laser treatment and stents are usually used for palliation. The tendency of margin positivity, even after luxurious resection beyond the visible tumour mass, adds to its insidious nature starting from symptoms that are very difficult to relate to the disease and to its treatment. 5 Conditions that contraindicate surgery are involvement of multiple lymph nodes, and involvement of unresectable mediastinal structures. Absolute contraindications include the presence of multiple positive lymph nodes, distant metastasis and more than half of tracheal involvement. 6

In this case, the patient was initially misdiagnosed as asthma. The case was diagnosed late and presented as a unique case with obstruction, for which emergency surgery was carried out successfully.

\section{REFERENCES}

1. Tracheal tumors: Background, anatomy, pathophysiology [Internet]. [cited 2018 Feb 1]. Available from: https:// emedicine.medscape.com/article/425904-overview 
2. Calzada AP, Miller M, Lai CK, Elashoff DA, Abemayor E, John MAS. Adenoid cystic carcinoma of the airway: A 30year review at one institution. Am J Otolaryngol 2012; 33: 226-31.

3. Gupta D, Singh I, Sakthivel P. Adenoid cystic carcinoma of trachea: A diagnostic and therapeutic challenge. Indian $J$ Otolaryngol Head Neck Surg 2016; 68:94-6.

4. Ahn Y, Chang H, Lim YS, Hah JH, Kwon TK, Sung MW, et al.
Primary tracheal tumors: Review of 37 cases. J Thorac Oncol 2009; 4:635-8.

5. Shimizu J, Oda M, Matsumoto I, Arano Y, Ishikawa N, Minato H. Clinicopathological study of surgically treated cases of tracheobronchial adenoid cystic carcinoma. Gen Thorac Cardiovasc Surg 2010; 58:82-6.

6. Macchiarini P. Primary tracheal tumours. Lancet Oncol 2006; 7:83-91.

.......... 